\title{
Seeing is believing: dental education benefits from developments in videoconferencing
}
IN BRIEF
- Videoconferencing is being used for teaching, distance consultation and providing specialist advice.
- One of the key advantages is the reduction in travel time and costs for tutors, students, patients and clinicians.
- The future appears to be in the use of Internet protocol (IP) links as they become increasingly cheap, secure and reliable.

\author{
P. A. Reynolds, ${ }^{1}$ K. A. Eaton ${ }^{2}$ and R. Mason ${ }^{3}$
}

Videoconferencing has been widely used to provide distant advice in many healthcare specialties across the world. However, videoconferencing has been further extended to support distance learning and has been evaluated through a number of educational projects. The use of the technology has been integrated as a core method of delivering training and education at all levels in dentistry. Advances in systems, services and standards have produced equipment and telecommunications links that are user-friendly, reliable, affordable and offer better quality image resolution. In addition, a wide range of peripheral devices can be connected to basic videoconferencing equipment to introduce a variety of teaching aids and material into a session. Projects such as PROVIDENT (Postgraduate Regional Online Videoconferencing in Dentistry) ${ }^{1}$ and DUET (Dental Undergraduate Education by Teleconferencing) ${ }^{2}$ have demonstrated that videoconferencing teaching sessions are as effective as traditional face-to-face lectures in both on-campus and off-campus teaching and reduce travel time and costs for both tutors and students. This paper describes how videoconferencing systems and services have developed and their benefits and applications in dentistry. It then goes on to describe the PROVIDENT and DUET projects as case studies.

\section{E-LEARNING IN DENTISTRY}

Section A: Teaching and technology

1. A description of the new technologies used in transforming dental education

2. Seeing is believing: dental education benefits from developments in videoconferencing

3. Webcasting: casting the web more widely

4. Top of the pops - CD-ROM and DVDs in dental education

Section B: Informatics: better informed

by systems and services

5. Better informed: an overview of health informatics

6. Better informed in clinical practice a brief overview of dental informatics

7. Digital clinical records and practice administration in primary dental care

Section C: Impact of e-learning in dental education

8. Remember the days in the old school yard: from lectures to online learning

9. An intricate web - designing and authoring a web-based course

10. The many faces of interaction

11. Supporting the learner and teacher online

12. Making a mark - taking assessment to technology

13. Continuing professional development and ICT: target practice

14. Assuring quality

Section D: A connected future

15. Nine years of DentEd: a global perspective

16. A vision of dental education in the third millenium

\section{INTRODUCTION}

In 1932 Flash Gordon ${ }^{3}$ used a voiceactivated, mobile, high quality, real time video system; its successor lives on in Star Trek. ${ }^{3}$ This may be the stuff of a fantasy world, but in reality videoconferencing has had a slow journey to acceptance in education and other fields.

The first commercial, analogue videoconferencing system appeared in 1963, but it took almost twenty years for the first digital version to be launched in 1982. ${ }^{3}$ For the next ten years videoconferencing suffered from being complex and expensive, with early digital systems resembling a studio setup with a large screen and separate, floor-standing cameras.

\footnotetext{
"Professor of Dental Education, Centre of Flexible Learning in Dentistry, King's College London Dental Institute, Floor 3, Strand Bridge House, 138-142 Strand, London, WC2 $1 \mathrm{HH} ;{ }^{2}$ Visiting Professor, UCL Eastman Dental Institute, 256 Gray's Inn Road, London, WC1X 8LD; ${ }^{3}$ Professor of Educational Technology, Institute of Educational Technology, The Open University, Walton Hall, Milton Keynes, MK7 6AA

${ }^{*}$ Correspondence to: Professor P. A. Reynolds Email:P.A.Reynolds@kcl.ac.uk
}

\section{Refereed Paper}

DOI: $10.1038 /$ bdj.2008.9

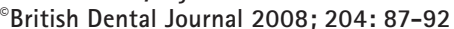

Since then costs have plummeted, systems have become far more reliable and have been transformed into integrated, easy-to-use, plug and play units or simple webcam and PC systems. Videoconferencing has also benefited from the availability of faster, more reliable digital telecommunications networks that ensure better quality transmission; and from a general reduction in the costs of calls. This is particularly true of the VoIP (voice over Internet protocol) systems, some of which are free to users globally, eg Skype ${ }^{\mathrm{TM}}$ (http://www.skype. com). Third generation mobile phones (3G) are capable of videoconferencing, but are expensive to use.

Videoconferencing can also be enhanced by its ability to link to other ICT components, be they hardware or software. PCs, laptops, CD-ROMs and VCRs can be connected to introduce a range of visual and oral teaching aids. Similarly, in what is known as applications sharing, software programmes running on a PC or laptop can be shared with all participants in a videoconference. Apart from PowerPoint presentations this can include word-processed and other material which can be worked on collaboratively. ${ }^{4}$ 


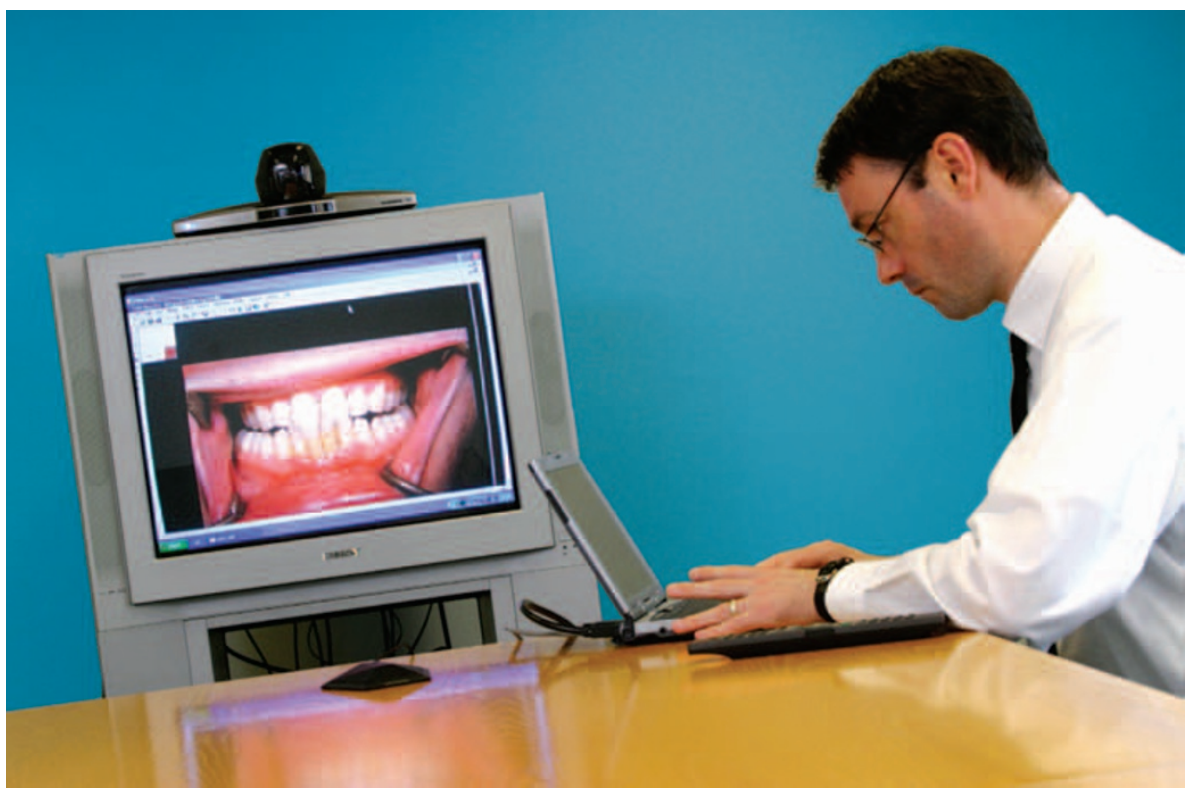

Fig. 1 A videoconference showing a close-up of a patient's teeth

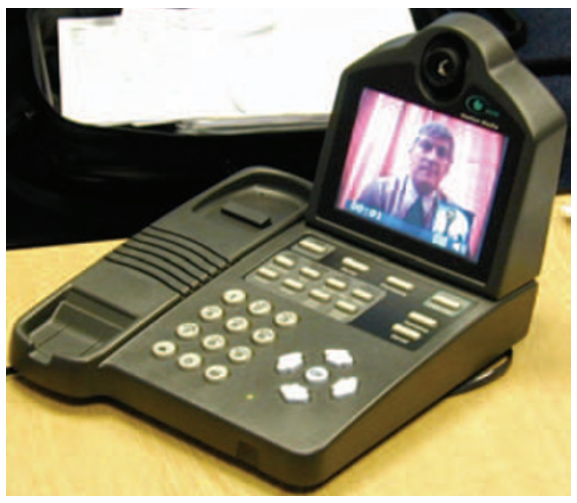

Fig. 2 A videophone

Specific videoconferencing peripherals have been developed such as visualisers that allow the display of three-dimensional objects, X-rays and paper-based items including charts and drawings. Whiteboards, on the other hand, enable tutors and students to create remote drawings or diagrams to demonstrate content. ${ }^{4,5}$

Because they are digital, modern videoconferencing systems can take a direct feed (input) from virtually any other digital system. This has important implications for dental and other healthcare professions. For example, students can be shown images from intra-oral cameras inserted into patients' mouths to show them dental conditions (Fig. 1). ${ }^{6}$

Standards have developed, too. Through the video coding standard, H.264, ${ }^{7}$ performance is being enhanced with transmission errors reduced and improved picture resolution through the virtual elimination of delay and jerkiness.

\section{SYSTEMS AND SERVICES}

A number of alternatives to a studio set-up for videoconferencing are now available. For individuals the choice is between videophones (Fig. 2), plug-in cards for PCs, or tabletop systems. All these devices incorporate a CODEC (videoconferencing card); network interfaces (IP and ISDN); a camera, microphone and speakers.

For other situations, ranging from small meetings of four to five people up to audiences in lecture theatres, the optimum solution entails choosing from a series of possible components which include: set-top boxes; control units using PC technology; standard TV sets, flat screens or twin screens; and incorporating videoconferencing into integrated audio-visual facilities.

Most of these 'room-based' systems offer analogue TV quality, but the advent of high definition TV (HDTV) is raising users' expectations of image quality. These are being satisfied by the launch of high definition videoconferencing (HDVC) which provides similar picture quality as its TV counterpart (Fig. 3). The resulting clarity and detail attainable will enhance the appeal of videoconferencing not just for medical education, but also for functions such as remote patient diagnosis and monitoring.

The advent of the integrated services digital network (ISDN), which is now virtually ubiquitous in the UK and many other countries, boosted the uptake of videoconferencing and has enabled the delivery of high quality links and reliable results. As Down ${ }^{8}$ has pointed out, ISDN has allowed 'the existing telephone infrastructure to carry digital signals.' The available bandwidth, on which the quality of picture depends, ranges from 64 Kbits to 2 Mbits. The most commonly used bandwidths for videoconferencing are $128 \mathrm{Kbits}$ (ISDN2), also known as two channel; and $384 \mathrm{Kbits}$ (ISDN6), also known as six channel.

A potential problem when ISDN2 links are used is that images may not be sharp. This problem can be overcome by sending the images in advance of a link, either as an email attachment, on a CDROM or as $35 \mathrm{~mm}$ slides, and projecting these images in the room/lecture theatre where the distant audience is present. ${ }^{9}$ The lecturer links via an ISDN2 videophone (Fig. 2). His/her image is projected on to one screen and the other images are projected on to a second screen. He/ she can see and hear the audience and they can see and hear him/her.

There are claims that Internet protocol (IP)-based broadband networks are more suited to carrying videoconferencing traffic, especially as most academics and many students have access to the Internet. However, until recently there was a drawback with IP-based links which was the lack of a guaranteed quality of service over the public IP network (the Internet) and over broadband: this has not been a problem with ISDN. According to some, ${ }^{10}$ IP-based videoconferencing has to contend with all data transmission, eg email, for the available bandwidth. The contention ratios are not stringent enough to cope with this mass of traffic and as IP is dynamically managed, data packets get dropped (some of the transmitted material is slowed down or lost). As videoconferencing is a real-time service, the loss of data or a delay in its transmission leads to a dramatic degradation in quality ranging from image fuzziness to incoherent speech. Provided that users accept these limitations, the use of VoIP as a low end IP-based solution is gaining momentum.

Internal IP networks are built to higher standards than the public one and thus quality of service can be guaranteed, internally across the campus and 


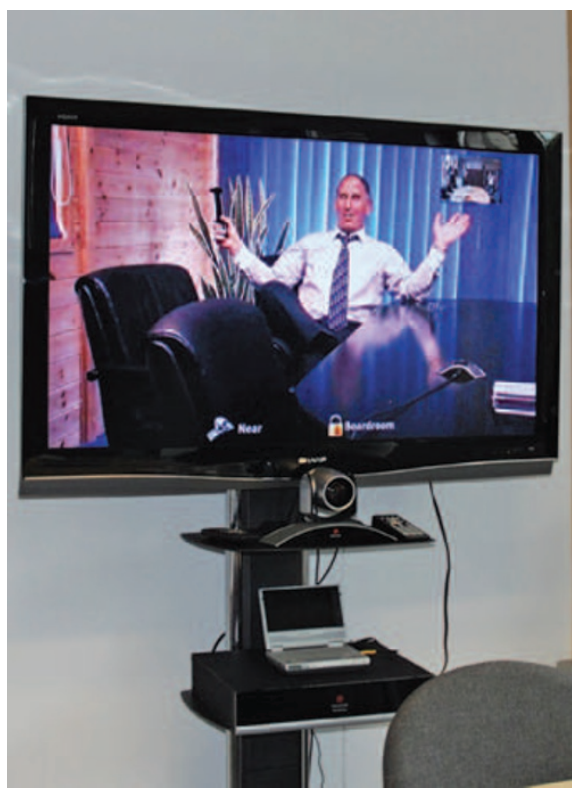

Fig. 3 High definition videoconferencing offers excellent image clarity

externally, to other UK academic institutions, over the JANET and SuperJANET networks. ${ }^{11}$

One method of overcoming the problem with the use of the Internet for videoconferencing can be to use it as a one-way link, as data can be buffered and lost packets requested before the broadcast is actually delivered. As such it is suitable for didactic learning, ie look and listen, but as it is not in real-time there can be no interaction. Known webconferencing users' computers can be connected to a server on the Internet or Intranet that has the content pre-loaded. Through it participants from dispersed locations such as homes or places of work can be reached. VoIP has been used to connect a lecturer from a distance where provided there was preservation of the audio, transient loss of video was not a considered a significant problem by the recipients. ${ }^{12}$

However, it is now possible to ensure that current videoconferencing systems can operate across ordinary business class Internet connections over the normal PSTN and enable individuals and groups to meet and share information. This allows users to enjoy IP's great advantage over public ISDN services - there are no call charges which, despite

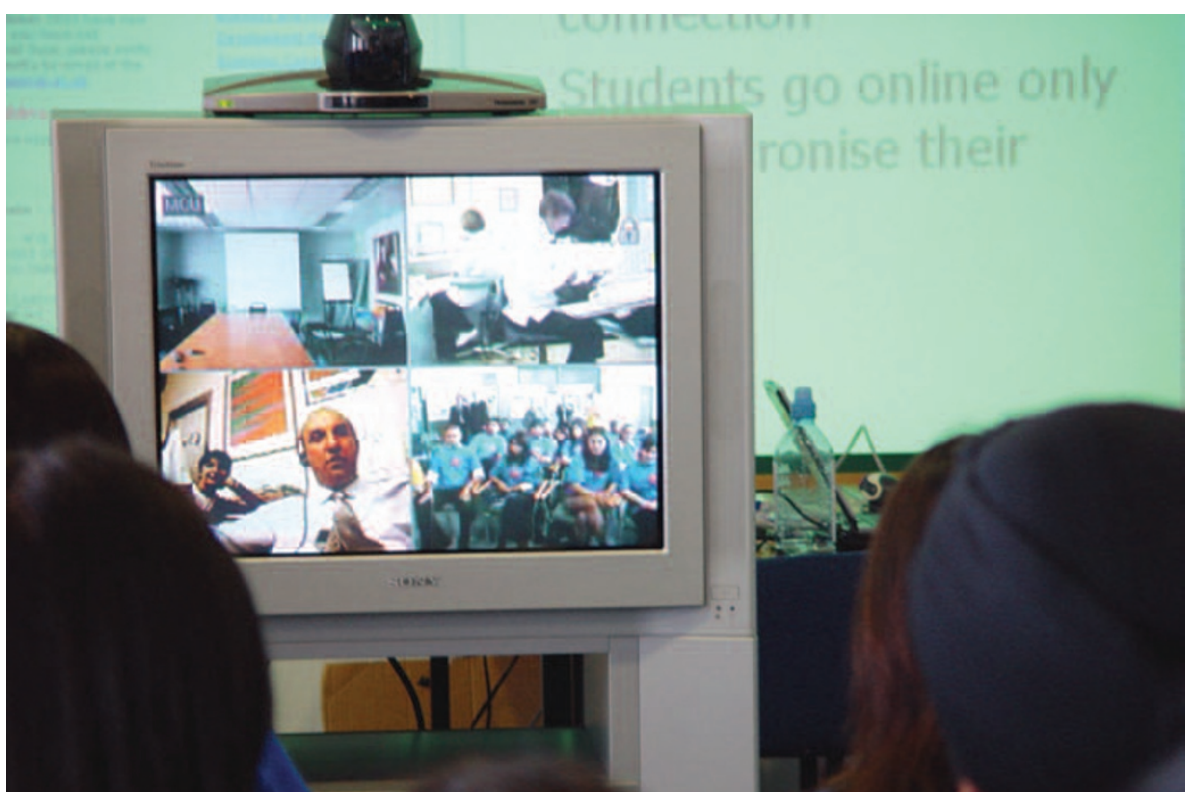

Fig. 4 A bridged link between four sites: a remote tutor and dental practice and two of the host school's campuses

ISDN's reliability and security, have limited its use for videoconferencing to large-scale events such as conferences and lectures rather than as a day-to-day communications tool. The latest IP and broadband developments, however, bring ISDN's operational benefits without the cost penalties. The normal broadband ADSL (asynschronous digital services lines) suffered from the drawback that the uplink speed (from user to network) is slower than the downlink (from network to user) making it unsuitable for the realtime needs of videoconferencing. With the latest flavour of broadband, SDSL (synchronous digital services lines), both speeds are the same.

The SDSL services now becoming available offer a minimum transmission speed of $384 \mathrm{Kbits}$, the optimum ISDN speed, and depending on the speed chosen, much higher image resolution of eg $1,024 \times 576$ pixels - six times that of traditional videoconferencing and higher than DVD quality.

There is no quality of service on the public Internet, a fact that may seem a disadvantage. However, as many of the latency and contention* problems can now be virtually eliminated, IP is fast becoming the prime communications medium and from August 2006 all calls in the UK that reach a carrier's network have become IP-based, proving that IP's previous service quality restrictions are a thing of the past. Voice over IP (VoIP) developments, such as the Skype service, also offer videoconferencing possibilities.

\section{BENEFITS AND APPLICATIONS OF VIDEOCONFERENCING}

Videoconferencing has been used for over 25 years to provide 'expert' opinions and advice to remote sites in a range of medical specialities and other areas of healthcare. ${ }^{13,14}$ From an educational perspective there are four main areas where videoconferencing has been of benefit: ${ }^{15}$

- Meetings: where bringing together participants who are geographically distributed is difficult, such as supervision of research students across campuses

- Teaching: to enable a specialist tutor to engage with a group of students (see cases studies - PROVIDENT - below)

- Management: committees across campuses or individuals who are off campus. Virtual graduations have been held by the Open University

- Interviews: either for jobs or as examination opportunities for oral components in research degrees.

In dentistry in the UK, videoconferencing has been used in this context 


\begin{tabular}{|c|c|}
\hline 1 & $\begin{array}{l}\text { How much time did it take you to prepare the lecture? Please compare this to a normal face to face } \\
\text { lecture (FT) }\end{array}$ \\
\hline 2 & $\begin{array}{l}\text { Was the technical assistance you had before and during the lecture adequate? Do you think you } \\
\text { could do it without an assistant when you are more experienced? (FT) }\end{array}$ \\
\hline 3 & $\begin{array}{l}\text { Overall, how would you compare this experience with the alternative of travelling to give the } \\
\text { lecture? (FT) }\end{array}$ \\
\hline 4 & Did you have any problems with sound/camera/audio-visual? (FT) \\
\hline 5 & $\begin{array}{l}\text { How did you feel about your ability to communicate with the remote participants? Were you able to } \\
\text { convey what you intended? (FT) }\end{array}$ \\
\hline 6 & How did you feel about the participants' response? (FT and $\mathrm{L}$ ) \\
\hline 7 & Was the question and answer session reasonably successful? (FT and L) \\
\hline 8 & Please make any other comments, especially notes to show other videoconference sites (FT) \\
\hline
\end{tabular}

to provide orthodontic diagnosis and advice reference, to improve communication between a dental surgery and a distant dental laboratory and to provide consultant advice in restorative dentistry from Aberdeen to the Highlands and Islands. ${ }^{16,17}$

Videoconferencing has been marketed predominantly on the basis that its use reduces travel time and costs, for both clinicians and patients. This is especially true for postgraduate and CPD courses where the participating 'students' already have some knowledge of the topics concerned and do not need intensive face-to-face, paperbased lectures. ${ }^{1,18}$ For such participants, learning in the workplace is far more useful, but they are frequently geographically spread and cannot always travel to a fixed location for lectures. By the same token many students throughout the world can benefit from on-going access to experts, experience and knowledge previously unavailable to them. Synchronous bridging between sites can bring together remote tutors, practices and schools, as seen in Figure 4.

As distance is no object, courses such as a number of Masters Degree programmes run by pioneering dental schools have been and are delivered effectively not only across the UK but in many other countries, provided the recipients can make a connection to an appropriate system in a domestic residence or an educational establishment (http://www. kcl.ac.uk/distancedentistry). ${ }^{19}$
Other dental education projects have used videoconferencing for a variety of purposes from teaching nurses in the Highlands and Islands to delivering courses worldwide. ${ }^{17,20}$ In the TELEDENT project its main uses were for large-scale lectures, case-based interactive tutorials and tutorials involving discussions where much of the knowledge resides with the learner. ${ }^{21}$ As one medium in an online ethics course, it has allowed debate in real-time on ethical dilemmas in training different cohorts of dental students. ${ }^{22}$

In dental education, videoconferencing has been used in both postgraduate and undergraduate courses, as illustrated by the PROVIDENT ${ }^{1,18}$ and DUET $^{2}$ projects.

\section{PROVIDENT project}

The aims of this off-campus project were:

- To assess the educational efficiency of videoconferencing in dental CPD

- To evaluate its practicality, costs and consumer satisfaction

- To evaluate the equipment used.

The project took place in 1998/1999, just before the General Dental Council introduced a CPD requirement of 250 hours over a five-year period for all UK dentists. Much of this continuing education takes place out of normal working hours and stretches teachers' ability and willingness to provide sufficient time, which could involve overnight stays

\section{CASE STUDIES IN DENTISTRY}

and/or travelling considerable distances, to deliver lectures and other activities.

Forty-one postgraduate videoconference teaching sessions were provided by 27 experienced academic staff from the (then) four London dental schools: The Eastman Dental Institute, King's College London (King's Denmark Hill and Guy's Hospital campuses) and The Royal London Hospital, with audiences attending one of eight postgraduate centres and two general dental practices in the then Thames Postgraduate Medical and Dental Education (TPMDE) Deanery. Two of the sessions were received in four and six centres respectively, the remainder were point-to-point. Low-cost desktop systems and ISDN2 connections were used.

\section{Training and educational content}

Before the first videoconference, a group of lecturers, postgraduate tutors and co-ordinators providing the technical support, were trained during a one-day course at the Open University. Training of individual speakers was then carried out locally by members of this group.

The educational content of the 41 sessions included prosthodontics, periodontics, endodontics, oral surgery, orthodontics, paediatric dentistry, oral imaging and infection control. Lectures, tutorials, interactive QCtA sessions and case studies were provided and involved the use of a wide variety of visual aids including $35 \mathrm{~mm}$ transparencies transmitted digitally and PowerPoint presentations (either given live or forwarded in advance digitally or as slides for projection). Three sessions also used the interactive whiteboards from the Microsoft NetMeeting programme. Radiographs were transmitted as live video camera images from a radiograph viewer or digitally in PowerPoint presentations. Images of flat artwork, live drawing, anatomical models, dental casts and instruments were transmitted using the integral document camera in a data projector.

\section{Assessment and results}

The project was assessed by questionnaire and structured follow-up interview. Both the participating dentists (audience) and the teachers completed a questionnaire. The teachers' 
questionnaire (Table 1) assessed difficulties with the medium and compared it with their experience of travelling to deliver equivalent teaching. Both presenter and audience were video-recorded and the recordings were assessed to evaluate technique. The teachers (presenters) were also interviewed by telephone on the day after they had delivered a videoconferenced teaching session. Only one of the sessions failed totally and this was for technical reasons. In five sessions sound quality was judged suboptimal by the presenter but not so poor that it prevented the completion of the sessions concerned.

The teachers found that:

- Their preparation time for videoconferencing teaching was virtually the same as for face-to-face lectures.

For 90\% of the sessions, preparation ranged from ten minutes to one hour

- The efficient use of time was noted by the teachers who were often able to videoconference during a short break from clinical duties at their hospitals

- Despite the general premonition that giving a videoconference would be stressful, most said that they felt surprisingly relaxed. The lack of human contact was felt to be a significant negative factor and most missed the opportunity for personal interaction.

The participating dentists (audiences):

- Were generally satisfied with their ability to communicate with the teachers

- Expressed reservations about the question and answer elements, although these were most affected by the technical problems especially during the multipoint links, which were often affected by poor sound quality

- Considered video quality generally satisfactory (poor visibility at viewing sites was due to poor selection of teaching material or poor equipment setup).

The video recordings of the audiences showed that they generally appeared passive, reflecting lack of contact. Presenters often felt that to improve the educational benefit, handouts or similar material should have been sent to the remote site in advance and that they needed to make very clear visual aids.

In the past, the provision of CPD for dentists in the UK has generally followed the episodic teaching model, which precludes development of longer-term educational relationships between teacher and learner. Videoconferencing can facilitate repeated interaction over long distances, favouring rapport building and reinforcement of learning. After the PROVIDENT project, some transmissions to dental practitioners in group practices were carried out. This proved popular but, given the small audience size, is inefficient for teachers. Nevertheless, if this method results in more regular contact it would be expected to improve the quality of the learning experience and is ideal for providing the type of interactive formal education which is most effective.

In a final report Mason ${ }^{23}$ outlined the conclusions that emerged from the analysis of the presenter feedback forms. These were:

- Technical assistance during the sessions will continue to be necessary for the future

- The lecturers consider the interaction with the audience to be somewhat inferior to that in a face-to-face situation, but not significantly so. It is somewhat more awkward, but there was general agreement that this could be improved with better sound systems and more familiarity

- The relatively poor sound quality remains the major technical limitation

- There is very strong endorsement of the educational model (not travelling) and the medium (almost as good as being there)

- The main issue for ongoing research and development of the model is what are its best uses? Regular lectures? Multi-site seminars?

As the incidence of technical problems reduced over the lifetime of the project, the number of negative comments from participants was markedly reduced. And where the sessions were free from technical hitches, the responses were overwhelmingly positive. Improvements would come from proper camera training and advanced information about the content of the session for questions to be structured to use the time successfully.

The interactive potential of the medium was rated most highly by the majority of participants. Many called for more informal, interactive sessions where they could 'butt in', work in small groups or indicate to the presenter at the beginning of the sessions what they wanted to know.

\section{DUET project}

In the DUET project, a videoconference link was provided to a dental practice in the Isle of Wight that allowed (and still allows) students to experience 'real-life' diagnosis and treatment through intraoral cameras integrated in the dentists' chairs. Undergraduates have until recently spent five years in a dental school environment and saw little of the real-life of a general dental practice; so the link to the practice gave them this first-hand experience.

The topics covered in the project included: medical emergencies, oral surgery, clinical practice, dental medicaments and pain control. Apart from remote lectures, seminars and training, the videoconference systems have also been used to provide pastoral care to students and during their elective visits.

The results of the assessment of the project indicated that almost all students $(98 \%)$ rated the sessions as good to very good, and over 95\% wanted more. Although local staff found the equipment complex, they valued the extra, direct experience gained by the students and additional cross-campus links saved travel time.

Teachers at the far (remote) end of videoconference links felt it was vital to have good timetabling, high quality equipment, and to test the links before each session. Their handling of the cameras improved with experience. In virtually all cases patients were willing to co-operate - they understood the value of excellent training for the next generation of dentists.

DUET's achievements can be summarised as:

- Weekly seminars for nearly 600 students over four years 
- Five major lectures delivered via videoconference links each year

- Student access to remote staff

- Experience of distance learning for teaching staff

- Student appreciation of the technology.

\section{CONCLUSIONS}

The PROVIDENT, DUET and other projects have demonstrated that videoconferencing has a key role to play in both undergraduate and CPD education in dentistry. It allows students to benefit from a wider range of expertise, knowledge and material than is normally possible and provides them with real-life experience of dental practice. The flexibility it offers means that both students and tutors are not constrained by geographical location and can enjoy these benefits no matter where they are based. However, the integration into curricula requires co-ordination and organisation, and with systems only just becoming reliable and affordable, this method of teaching has still to be established. Videoconferencing may have been thought of as one of the killer applications of our time, but its use and integration in dental education is still emerging.

1. Eaton K A, Francis C A, Odell E W, Reynolds PA, Mason R D. Participating dentists' assessment of the pilot regional online videoconferencing in dentistry (PROVIDENT) project. Br Dent J 2001; 191: 330-334.

2. Reynolds.PA. Dental undergraduate education by teleconferencing (DUET): experiences over 4 years. Technol Health Care 2001; 9: 321.

3. McMaster S. Management policy for use of videoconferencing facilities. Nottingham: Questmark Ltd, 2002.

4. Stephens C D. Forget the sailboard - let's go whiteboarding! Dent Update 2000; 27: 236-240.

5. Magee R, Wheeler S. Distance learning and new convergent technologies. RATIO Project, University of Plymouth, 1997.

6. Reynolds P A, Harper J. Dental school teaches and treats via videoconferencing. The Dental Institute, King's College London, 2000.
7. ITU Telecommunication Standardization Sector Advanced video coding for generic audiovisual services. ITU-T recommendation H.264. ITU, May 2003.

8. Down P H. Introduction to videoconferencing. Video Technology Advisory Service, University of Newcastle, 2001.

9. Eaton KA. The integrated use of videophones and $C D$ ROMs to overcome bandwidth limitations during video-conferenced lectures. Technol Health Care 2001; 9: 320

10. Snow M D, Canale E, Quail G.Teledentistry permits distant, cost-effective specialist dental consultations for rural Australians. J Telemed Telecare 2000; 6 (Suppl 1): 216.

11. JANET. About Janet. http://www.ja.net/about/ index.html. Accessed 15 November 2007.

12. Reynolds P A, Millar B. Feedback Residential Course MClinDent. King's College London Dental Institute, July 2006

13. Elford R. Telemedicine activities at the Memorial University of Newfoundland: a historical review, 1975-1997. Telemed J 1998; 4: 207-224.

14. Edelman $G$, Hall A. Innovation and partnership: the voluntary sector contribution. Int J Lang Commun Disord 1998; 33: 10-15.

15. Mason R, Rennie F. Elearning: the key concepts. London: Routledge, 2006.

16. Cook J, Austen G, Stephens C D. Videoconferencing: what are the benefits for dental practice? $\mathrm{Br}$ Dent J 2000; 188: 67-70.

17. Steed M. Evaluation of a teledental PC videoconferenced link in the delivery of a restorative dentistry service to remote practices in Scotland. Proceedings of the 7 th International Conference on Telemedicine and Telecare. London, 1999.

18. Odell E W, Francis C A, Eaton K, Reynolds PA, Mason R D. A pilot study of videoconferencing for postgraduate continuing education in dentistry in the UK - the teachers' view. Eur J Dent Educ 2001; 5: 113-119.

19. King's College London. New online degree in clinical dentistry. Kings College London News 2004 September 18. http://www.kcl.ac.uk/phpnews/wmview. php?ArtID=672 Accessed 8 November 2004.

20. Chen J W, Hobdell M H, Dunn K, Johnson K A, Zhang J. Teledentistry and its use in dental education. J Am Dent Assoc 2003; 134: 342-346.

21. Cook J, Grigg P, Stephens C, Williams J, Vowles R. TeleDent Project. Institute for Learning and Research Technology, University of Bristol, 1998.

22. Louca C, Reynolds P A, Coates R, Paganelli C, Cox $\mathrm{M}$ J. Comparison of online activities between four different cohorts of dental students undertaking an international introductory ethics and professionalism course. (Poster 7). Proceedings of the Association of Dental Educators of Europe (ADEE) 32nd Annual Meeting. 30 Aug-2 September 2006, Krakow, Poland. Abstract p29.

23. Mason R D. The PROVIDENT project final report. Milton Keynes: Open University, 2001.

\section{GLOSSARY OF TERMS}

CD-ROM - Compact disc read only memory, a device for storing and accessing large amounts of data in both text and visual forms DUET - Dental Undergraduate Education by Teleconferencing, a project

ICT - Information and communications technologies; a generic term for computing and telecommunications technologies

H.264 - A video coding standard that offers better utilisation of network capacity by reducing bandwidth requirements by up to $50 \%$ through improved compression techniques HDTV - High definition digital television HDVC - High definition videoconferencing CODEC - CODer/DECoder. Equipment that converts video digital signals into bandwidth for transmission across an analogue network IP - Internet protocol, the standard that governs data-packet forwarding across the Internet

ISDN - Integrated services digital network. A public network service for the transmission of digitised voice, video and data signals over the existing public analogue telephone network

JANET - Joint academic network. The UK education and research communities' backbone network. SuperJANET is an upgraded, faster version

NetMeeting - Microsoft's software conferencing system that allows users to communicate and collaborate in real-time during a videoconference PROVIDENT - Postgraduate Regional Online Videoconferencing in Dentistry VCR - Video cassette recorder 